\title{
Effects of Shen-Fu Injection on the Expression of T-Cell-Specific Transcription Factors T-bet/Gata-3 in Porcine Postresuscitation Lung Injury
}

\author{
Wei Gu, ChunSheng Li, WenPeng Yin, XiaoMin Hou, and Da Zhang \\ Department of Emergency Medicine, Beijing Chaoyang Hospital, Capital Medical University, Beijing 100020, China \\ Correspondence should be addressed to ChunSheng Li; banditgu@hotmail.com
}

Received 28 November 2012; Accepted 17 February 2013

Academic Editor: Rocio De la Puerta

Copyright (C) 2013 Wei Gu et al. This is an open access article distributed under the Creative Commons Attribution License, which permits unrestricted use, distribution, and reproduction in any medium, provided the original work is properly cited.

\begin{abstract}
Shen-Fu injection (SFI) derived from the ancient traditional Chinese medicine. In this study, the effects of SFI on the expression of T-bet/GATA-3 and its potential mechanisms causing the shift of T cells from Th2 to Th1 on postresuscitation lung injury were examined in a porcine model of cardiac arrest. 30 pigs were randomly divided into SHAM $(n=6)$ and three return of spontaneous circulation (ROSC) groups ( $n=8$ per group); 24 pigs were subjected to $8 \mathrm{~min}$ of electrically induced cardiac arrest and $2 \mathrm{~min}$ of basic life support, which received central venous injection of Shen-Fu (SFI), epinephrine (EP) or saline (SA). After successful ROSC, 18 surviving pigs were sacrificed at $24 \mathrm{~h}$ after ROSC ( $n=6$ per group). The levels of serum and lung tissue interleukin (IL) -4 and interferon (IFN)- $\gamma$ were measured by ELISA, and the protein and mRNA levels of GATA-3 and T-bet in the lung tissue were determined by western blotting and quantitative real-time polymerase chain reaction, respectively. Compared with the EP and SA groups, SFI treatment reduced the levels of IL-4 $(P<0.05)$, increased levels of IFN- $\gamma(P<0.05)$, and induced T-bet mRNA upregulation and GATA-3 mRNA downregulation $(P<0.05)$. SFI attenuated lung injury and regulated lung immune disorders. Therefore, SFI could protect postresuscitation lung injury by modulating a Th1/Th2 imbalance.
\end{abstract}

\section{Introduction}

Cardiac arrest (CA) represents the most severe shock state, during which the delivery of oxygen and metabolic substrates is abruptly halted. Increased oxygen debt leads to endothelial activation and results in the systemic release of pro- and anti-inflammatory mediators [1], such as tumour necrosis factor- $\alpha$ (TNF- $\alpha$ ), which promotes immune suppression and systemic inflammation [2], thereby contributing to postresuscitation lung injury. However, the mechanisms responsible for postresuscitation lung injury are not well understood. Several other mechanisms have been not only hypothesized but shown to be involved, including oxygen-derived-free radicals, ischemia/reperfusion, and apoptosis [3-5].

The balance of T helper type 1 (Th1) and T helper type 2 (Th2) cells is important in regulating immune function and inflammatory response. Thl lymphocytes produce IFN- $\gamma$ and interleukin (IL)-2, favouring cell-mediated immunity; Th2 lymphocytes secrete IL-4, IL-5, IL-10, and IL-13, favouring humoral immunity [6]. As Th1 and Th2 cells are differentiated from common $T$ precursor cells, their differentiation requires the activity of specific transcription factors, including T-bet and GATA-3 $[6,7]$. Thus, changes in the expression levels of GATA- 3 and T-bet contribute to an imbalance in Th1 and Th2 cells. Protective immunity is dependent on the proper balance of Th1 and Th2 cells [8]. Alterations in the correct balance of Th1/Th2 cells are associated with a series of immune and inflammatory diseases, such as burn or trauma, acute myocardial infarction, heart failure, and severe sepsis [9-13].

Shen-Fu injection (SFI) derived from the ancient traditional chinese medicine and mainly composed of Ginsengand Fuzi extracts (Yảan Sanjiu Pharmaceutical Co., Ltd., China). Its quality is controlled strictly according to the standard of China Ministry of Public Health, and fingerprint technology was adopted in the process of production to ensure its quality. Some studies have proved that SFI had protective effect on acute lung injury induced by endotoxin and shock [14, 15]. Our previous work also showed that SFI can attenuate 
postresuscitation lung injury by modulating apoptosis [5]. However, the other mechanisms responsible for the lung protective effects of SFI are unknown. Therefore, in this study, we established a swine model of CA, in which we examined the effects of SFI on the expression of T-bet and GATA-3, and found that SFI after ROSC could reduce postresuscitation lung injury by modulating lung Th1/Th2 imbalance.

\section{Materials and Methods}

The study was conducted with the approval of The Capital Medical University Institutional Animal Care Committee, and all animals received treatments in compliance with the National Research Council's 1996 Guide for the Care and Use of Laboratory Animals.

2.1. Animal Preparation. Thirty inbred male Wuzhishan miniature pigs (12-14 months of age, $30 \pm 2 \mathrm{~kg}$ ) were used in this study. Pigs were randomly divided into 4 groups, resuscitation groups: Shen-Fu injection (SFI), epinephrine (EP), saline (SA) ( $n=8$, per group), and sham operation $(\mathrm{SHAM})(n=6)$. Animals were fasted overnight but were allowed free access to water. After premedication with $0.5 \mathrm{mg} / \mathrm{kg}$ intramuscular midazolam, anesthesia was induced by ear vein injection of propofol $(1.0 \mathrm{mg} / \mathrm{kg})$ and maintained in a surgical plane of anesthesia with intravenous infusion of pentobarbital $(8 \mathrm{mg} / \mathrm{kg} / \mathrm{h})$ [15]. Heart rate and electrocardiogram measurements were monitored using a four-channel physical recorder (BL-420F Data Acquisition \& Analysis System; Chengdu TME Technology Co, Ltd, China). A cuffed $6.5 \mathrm{~mm}$ endotracheal tube was advanced into the trachea. Animals were mechanically ventilated with a volume-controlled ventilator (Servo 900C; Siemens, Germany) with a tidal volume of $15 \mathrm{~mL} / \mathrm{kg}$ and $\mathrm{FiO}_{2}$ of 0.35 using a tidal volume of $15 \mathrm{~mL} / \mathrm{kg}$ and a respiratory frequency of $12 / \mathrm{min}$. An angiographic catheter was inserted from the femoral artery into the aortic arch to measure aortic pressure. The electrocardiogram and all hemodynamic parameters were monitored with a patient monitoring system (M1165; Hewlett-Packard, Palo Alto, CA).

2.2. Experimental Protocol. After surgery, the animals were allowed to equilibrate for $30 \mathrm{~min}$ to achieve a stable resting level. The temporary pacemaker conductor was inserted into the right ventricle through the right sheathing canal and connected to an electrical stimulator (GY-600A; Kaifeng Huanan Equipment Co., Ltd., China) programmed in the S1S2 mode (300/200 ms), 40 V, 8:1 proportion, and $10 \mathrm{~ms}$ step length to provide a continuous electrical stimulus until ventricular fibrillation (VF) [16]. VF was defined as an electrocardiogram showing waveforms corresponding to VF and a rapid decline in mean aortic pressure toward zero [17]. Ventilation was stopped while inducing VF, and ventilation was withheld for the entire 8-minute duration of VF arrest. After $8 \mathrm{~min}$ of VF, manual CPR was carried out at a frequency of 100 compressions/min with mechanical ventilation at an $\mathrm{FiO}_{2}$ of $100 \%$ and a compression-to-ventilation ratio of $30: 2$. The quality of chest compressions was controlled by a HeartStart MRx Monitor/Defibrillator with Q-CPR (Philips
Medical Systems, Best, Holland) [16]. After 2 minutes of $\mathrm{CPR}$, pigs were randomized divided into 3 groups, then receive, respectively, central venous injection of Shen-Fu injection $(1.0 \mathrm{~mL} / \mathrm{kg})(\mathrm{SFI})$, epinephrine $(0.02 \mathrm{mg} / \mathrm{Kg})(\mathrm{EP})$ and saline (SA). If the spontaneous circulation was not restored, defibrillation was attempted once using a diphase $150 \mathrm{~J}$. If spontaneous circulation was still not achieved, CPR was continued for a further 2 minutes and defibrillation was attempted once more. The same procedure without CA initiation was achieved in the SHAM group, including induction of anaesthesia, electrode positioning, mechanical respiration, 8 mins ventilation withheld, and monitoring of physiological parameters.

ROSC was defined as 10 consecutive min of maintenance of systolic blood pressure at $50 \mathrm{~mm} \mathrm{Hg}$. If spontaneous circulation was not restored within $30 \mathrm{~min}$, we regarded the animal as dead [17]. The survival animals were euthanized with $10 \mathrm{~mL}$ of $10 \mathrm{~mol} / \mathrm{L}$ potassium chloride intravenously at $24 \mathrm{hrs}$ after resuscitation. The left external jugular vein was cut open, the catheter was put into the right common carotid artery and normal saline was infused through the catheter smoothly into the artery until the blood became clear, then the chest was opened, and the right middle lung lobe was separated and obtained.

2.3. Measurements. Arterial blood samples were taken at baseline, 0, $30 \mathrm{~min}, 2$, 6 hours after ROSC; furthermore, venous blood samples were taken at baseline, $30 \mathrm{~min}, 6,12$, and 24 hours after ROSC. Arterial blood gas was examined (GEM Premier 3000 blood gas analyzer, Instrumentation Laboratory, Lexington, MAs). Some lung specimens were preserved in $4 \%$ paraformaldehyde to observe pathologic changes for light microscope and transmission electron microscopy, whereas others were stored at $-80^{\circ} \mathrm{C}$ for enzymelinked immunosorbent assay (ELISA), quantitative real-time PCR, and western blotting.

2.4. The Measurement of Lung W/D. The left upper lobe of the lungs was obtained, the blood and water of the lung surface were removed by using absorbent paper to get the "wet" weight (W), and then the wet lung was put into stoving chest at $70^{\circ} \mathrm{C}$ for $72 \mathrm{hrs}$ to get the "dry" weight (D). The ratio of the wet lung to the dry lung was calculated to assess tissue edema.

2.5. BALF Total Cell and PMN Counts. The pigs were anesthetized again at $24 \mathrm{~h}$ after ROSC and provided with a plastic cannula inserted into the trachea. BALF was performed with three aliquots of $5 \mathrm{~mL}$ PBS (pH7.2) instilled up to a total volume of $15 \mathrm{~mL}$ and withdrawn three times each. The recovery rate of BALF was $95 \%$. BALF samples were centrifuged $\left(400 \times \mathrm{g}, 4^{\circ} \mathrm{C}\right)$ for $5 \mathrm{~min}$. The sediment cells were resuspended in $100 \mu \mathrm{L}$ PBS. The total BALF cells were counted double blindly using a hemocytometer followed by the differential counting of leukocytes (Giemsa staining; two counts per slide, 300 cells per count).

2.6. Th1 and Th2 Related Cytokines Analyses. IL-4 (6R019) and IFN- $\gamma$ (6R082) in the serum and lung tissue were measured by ELISA according to the manufacturers'instructions 
(Sunbio Biotech Co. Ltd., China). $100 \mathrm{mg}$ lung tissue and $2 \mathrm{~mL}$ vein blood were, respectively, centrifuged at $35,000 \times \mathrm{g}$ for $10 \mathrm{~min}$ at $4^{\circ} \mathrm{C}$, supernatants and centrifuged blood were collected and immediately frozen at $-80^{\circ} \mathrm{C}$ for ELISA, microtitration plates were coated overnight with $50 \mathrm{uL}$ of a $10 \mathrm{ug} / \mathrm{mL}$ solution of Biotin anti- IL-4 and INF- $\gamma$ in carbonate buffer $\mathrm{pH}$ 9.6. After blocking and washing, the sample was incubated for $30 \mathrm{~min}$ at room temperature. Plates were washed and added with $50 \mu \mathrm{L}$ HRP, then, incubated for $30 \mathrm{~min}$ again at room temperature, and subsequently, the plates were washed again. Each well was added with $50 \mu \mathrm{L}$ color liquid, gently mixed for 30 seconds, and incubated for 15 minutes at room temperature. The reaction was stopped after $15 \mathrm{~min}$ by the addition of $50 \mu \mathrm{L}$ stop solution. The absorbance at $450 \mathrm{~nm}$ was measured after 30 min using an ELISA plate reader. Cytokine concentrations were obtained from a standard curve. Duplicate readings were obtained for all samples and the means were calculated.

2.7. Western Blot Analysis of GATA-3 and T-bet. A 150$\mathrm{mg}$ frozen lung sample was homogenized in $2 \mathrm{~mL}$ of icecold buffer. The lung tissues were homogenized and then centrifuged at $12,000 \mathrm{r} / \mathrm{min}$ for $10 \mathrm{~min}$ at $4^{\circ} \mathrm{C}$. A total of $100 \mu \mathrm{g}$ of protein was loaded onto $10 \%$ SDS-PAGE gel in each sample. Western blotting was performed with the membranes blocked for $2 \mathrm{~h}$ with $5 \%$ nonfat milk and then incubated with the primary antibodies (diluted overnight at $4^{\circ} \mathrm{C}$ ): GATA-3, 1:500 (ab113519; Abcam Biotechnology, UK); T-bet, 1:200 (sc-21003; Santa Cruz Biotechnology, USA); and GAPDH, 1: 250 (Santa Cruz Biotechnology, USA). Blots were blocked and incubated at $4^{\circ} \mathrm{C}$ overnight with the specific primary antibody. The immunoreactive bands were visualized on film and scanned. The data were analysed by Image Pro Plus (Version 4.1, Media Cybernetics). The quantitative data from Western blot bands were expressed as the target protein OD/GAPDH OD ratio.

2.8. Quantitative Real-Time PCR Assay for GATA-3 and Tbet. Total RNA was extracted from 50 to $100 \mathrm{mg}$ of lung tissue according to the protocol described for the BioEasy SYBR Green I Real-Time PCR Kit Manual (Bo Ri Technology Co., Ltd., China). Preincubation was performed at $95^{\circ} \mathrm{C}$ for $2 \mathrm{~min}$, followed by amplification in 45 cycles at $95^{\circ} \mathrm{C}$ for $20 \mathrm{~s}, 59^{\circ} \mathrm{C}$ (GATA-3 and T-bet), $72^{\circ} \mathrm{C}$ for $30 \mathrm{~s}$, and finally, during slow heating up, $72^{\circ} \mathrm{C}$ for $10 \mathrm{~min}$. After the amplification, melting curve analysis with a temperature gradient from $65^{\circ} \mathrm{C}$ to $95^{\circ} \mathrm{C}$ was recorded every $0.5^{\circ} \mathrm{C}$ (hold for $5 \mathrm{~s}$ ). The primer sequences of the expected PCR products were as follows: for GATA-3 (240 bp), Forward primer: $5^{\prime}$ TGCGGGCTCTACCACAAAAT- $3^{\prime}$ and reverse primer: $5^{\prime}$ TAACCCGAGTAAAATGTGC- $3^{\prime}$; for T-bet (118 bp), forward primer: $5^{\prime}$-ACAAACCCGATATGGCTGAGA- $3^{\prime}$ and reverse primer: $5^{\prime}$-CCTGCTTGCTTCTCCTGTTC- $3^{\prime}$; and for GAPDH, Forward primer: $5^{\prime}$-CCATCACTGCCACTCAGAAGACT- $3^{\prime}$, and Reverse primer: $5^{\prime}$-GTCAGATCCACAACGGATACATTG- $3^{\prime}$. Relative quantification is generally calculated with the $2^{-\Delta \Delta C T}$ formula by the comparative $\mathrm{Ct}$ method, the copy number of the target gene $2^{-\Delta \Delta \mathrm{CT}}=$ $2^{-(\triangle \mathrm{CT} \text { target gene }-\triangle \mathrm{CT} \text { GAPDH gene) }}[17]$.
TABLE 1: Resuscitation outcome.

\begin{tabular}{|c|c|c|c|}
\hline Group & $\begin{array}{l}\text { Number of } \\
\text { shock }\end{array}$ & $\begin{array}{c}\text { Energy of shock } \\
(\mathrm{J})\end{array}$ & $\begin{array}{c}\text { Time to ROSC } \\
\text { (min) }\end{array}$ \\
\hline SA & $5.5 \pm 2.5$ & $795 \pm 375.68$ & $10.0 \pm 3.79$ \\
\hline $\mathrm{EP}$ & $2.75 \pm 1.66^{*}$ & $312.7 \pm 134.28^{* *}$ & $6 \pm 2.17^{* *}$ \\
\hline SFI & $2.61 \pm 1.03^{* \boldsymbol{\Delta}}$ & $\begin{array}{c}332.5 \pm \\
168.39^{* * \boldsymbol{\Lambda}}\end{array}$ & $5 \pm 1.69^{* * \boldsymbol{\Delta}}$ \\
\hline
\end{tabular}

${ }^{*} P<0.05,{ }^{* *} P<0.01$ versus SA (one-way repeated-measures ANOVA); $\boldsymbol{\Delta} P>0.05$, versus EP (student's $t$ test).

2.9. Statistical Analysis. The experimental data were analyzed by SPSS 17.0 (SPSS Inc., Chicago, IL, USA). The results are expressed as mean $\pm \mathrm{SD}$, and Student's $t$ test was used for comparisons between two groups. Differences at different time points within groups were compared with repeatedmeasures ANOVA. A two-tail value of $P<0.05$ was considered significant.

\section{Results}

18 of 24 animals were successfully resuscitated. By comparison, the number of electric shock, defibrillation energy, and time to ROSC were significantly lower in EP and SFI group than that in SA group $(P<0.05$, Table 1$)$, but there was no difference in EP and SFI groups $(P>0.05$, Table 1$)$.

3.1. Blood Gas Analysis. $\mathrm{PH}$ and $\mathrm{PaO}_{2}$ in the post-ROSC group decreased more significantly than those in the shamoperated group within post-ROSC $6 \mathrm{~h}$ (Table 2), Furthermore, $\mathrm{PH}$ and $\mathrm{PaO}_{2}$ in the SFI group increased more significantly than those in the EP and SA groups (Table 2).

3.2. Ultrastructural Changes in Lung Tissue. By light microscopy, lung tissue structure appeared normal in the SHAM group (arrows) (Figure 1(a)), whereas it was severely damaged in the EP and SA groups. The alveolar cavity was narrow, the alveolar wall became thick, and the capillary of alveolar wall dilated. There was obvious blood and edema fluid exudation in alveolar cavity. There were also exfoliated epithelial cells and neutrophil infiltration in alveolar cavity. (Figures 1(b) and 1(c)). Congestion, inflammatory cell infiltration, and edema of the lung tissue were considerably alleviated with SFI treatment (Figure 1(d)). Using electron microscopy, normal type 2 epithelial cells structure was observed in the SHAM group (arrows) (Figure 1(e)). Epithelial cells turned into necrosis and disintegration, and the lung tissue exhibited progressive, severe deterioration in the EP and SA groups $24 \mathrm{~h}$ after cardiac resuscitation (arrows) (Figure 1(f)). The lamellar body (characteristic structures of type II epithelial cells, which produce pulmonary surfactant to reduce alveolar surface tension) of type 2 epithelial cells cracked (arrows) (Figure $1(\mathrm{~g})$ ). Animals treated with SFI exhibited minimal type 2 epithelial cells structure damage in the lung tissue (Figure 1(h)). 
TABLE 2: Changes of arterial blood gas $\left(\mathrm{PH}, \mathrm{PaO}_{2}, \mathrm{PaCO}_{2}\right.$, and $\left.\mathrm{PaO}_{2} / \mathrm{FiO}_{2}\right)$ at baseline, $0,30 \mathrm{~min}, 2 \mathrm{~h}$, and $6 \mathrm{~h}$ after ROSC in SHAM, SA, EP and SFI groups (mean $\pm \mathrm{SD})$.

\begin{tabular}{|c|c|c|c|c|c|}
\hline Group & baseline & ROSC $0 \mathrm{~min}$ & ROSC $30 \mathrm{~min}$ & ROSC $2 \mathrm{~h}$ & ROSC $6 \mathrm{~h}$ \\
\hline \multicolumn{6}{|l|}{$\mathrm{PH}$} \\
\hline SHAM & $7.42 \pm 0.05$ & $7.44 \pm 0.03$ & $7.46 \pm 0.11$ & $7.38 \pm 0.09$ & $7.41 \pm 0.14$ \\
\hline SA & $7.42 \pm 0.12$ & $7.05 \pm 0.17^{* *}$ & $7.11 \pm 0.19^{* *}$ & $7.19 \pm 0.23^{*}$ & $7.31 \pm 0.11$ \\
\hline $\mathrm{EP}$ & $7.39 \pm 0.12$ & $7.01 \pm 0.29^{* *}$ & $7.03 \pm 0.18^{* *}$ & $7.08 \pm 0.27^{*}$ & $7.28 \pm 0.19$ \\
\hline SFI & $7.41 \pm 0.21$ & $7.02 \pm 0.13^{* *}$ & $7.23 \pm 0.24^{* * \boldsymbol{\Lambda}}$ & $7.28 \pm 0.27^{* \boldsymbol{\Delta}}$ & $7.38 \pm 0.27$ \\
\hline \multicolumn{6}{|l|}{$\mathrm{PaO}_{2}$} \\
\hline SHAM & $100.9 \pm 6.4$ & $93.3 \pm 5.7$ & $97.5 \pm 4.1$ & $98.9 \pm 7.1$ & $104.2 \pm 6.1$ \\
\hline SA & $98.4 \pm 4.9$ & $41.4 \pm 6.1^{* *}$ & $59.8 \pm 3.1^{*}$ & $64.9 \pm 2.8^{*}$ & $75.7 \pm 2.1$ \\
\hline $\mathrm{EP}$ & $100.4 \pm 3.8$ & $38.4 \pm 5.3^{* *}$ & $53.5 \pm 2.8^{*}$ & $58.1 \pm 3.6^{*}$ & $69.7 \pm 3.7$ \\
\hline SFI & $98.4 \pm 4.9$ & $37.8 \pm 5.2^{* *}$ & $60.1 \pm 4.9^{*}$ & $72.6 \pm 2.4^{* \mathbf{\Delta}}$ & $83.1 \pm 2.6^{\boldsymbol{\Lambda}}$ \\
\hline \multicolumn{6}{|l|}{$\mathrm{PaCO}_{2}$} \\
\hline SHAM & $36.1 \pm 2.6$ & $35.2 \pm 1.9$ & $34.3 \pm 3.7$ & $37.1 \pm 2.1$ & $38.1 \pm 1.9$ \\
\hline SA & $35.9 \pm 1.4$ & $49.7 \pm 4.4^{*}$ & $44.7 \pm 3.8$ & $42.2 \pm 2.5$ & $37.5 \pm 3.6$ \\
\hline $\mathrm{EP}$ & $36.4 \pm 2.8$ & $53.7 \pm 3.6^{*}$ & $47.7 \pm 4.1$ & $46.1 \pm 3.3$ & $39.8 \pm 2.9$ \\
\hline SFI & $37.4 \pm 1.7$ & $55.2 \pm 3.6^{*}$ & $43.6 \pm 3.1$ & $44.1 \pm 4.2$ & $38.8 \pm 3.5$ \\
\hline \multicolumn{6}{|l|}{$\mathrm{PaO}_{2} / \mathrm{FiO}_{2}$} \\
\hline SHAM & $293.2 \pm 35.6$ & $278.1 \pm 16.2$ & $282.8 \pm 19.5$ & $292.8 \pm 11.7$ & $299.7 \pm 9.9$ \\
\hline SA & $299.5 \pm 22.1$ & $114.8 \pm 18.7^{* *}$ & $178.5 \pm 19.3^{*}$ & $181.4 \pm 16.8^{*}$ & $207.6 \pm 8.5$ \\
\hline $\mathrm{EP}$ & $302.1 \pm 17.2$ & $107.8 \pm 15.9^{* *}$ & $155.5 \pm 13.4^{*}$ & $176.1 \pm 19.8^{*}$ & $200.1 \pm 7.9$ \\
\hline SFI & $297.5 \pm 18.3$ & $109.8 \pm 17.6^{* *}$ & $188.5 \pm 9.8^{*}$ & $209.6 \pm 14.1^{* \boldsymbol{\Delta}}$ & $247.6 \pm 9.1^{\wedge}$ \\
\hline
\end{tabular}

The values represent mean $\pm \operatorname{SE}(n=6){ }^{*} P<0.05$ and ${ }^{* *} P<0.01$ versus SHAM; ${ }^{\mathbf{A}} P<0.05$ versus EP and $S A$.

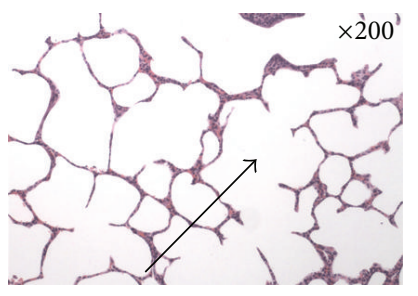

(a)

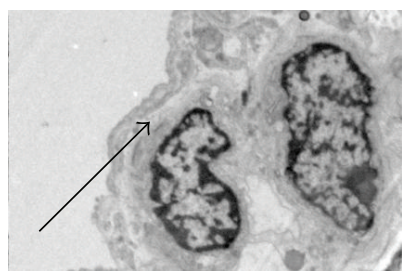

(e)

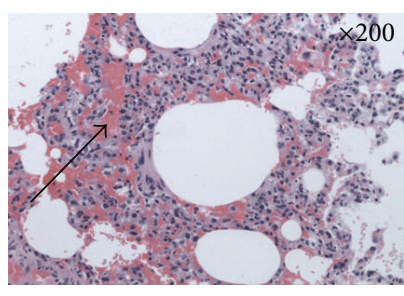

(b)

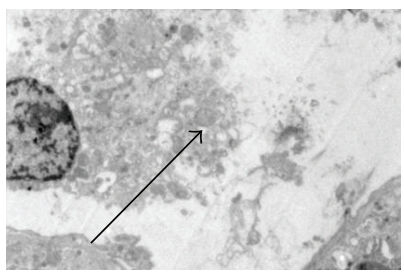

(f)

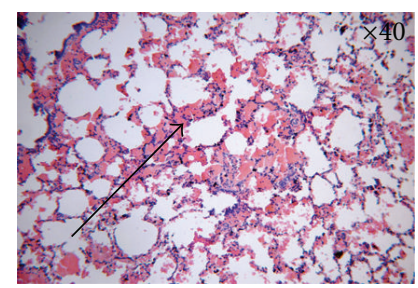

(c)

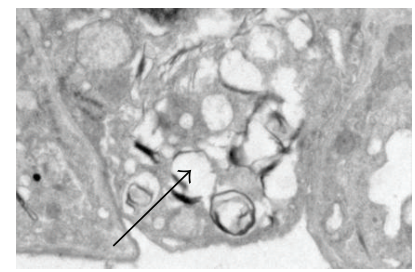

(g)

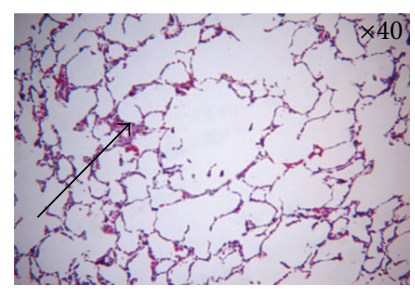

(d)

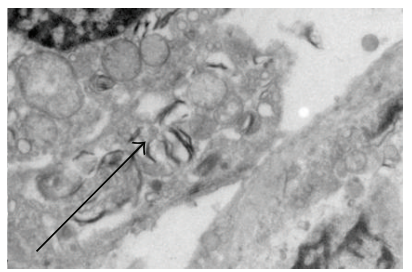

(h)

FIGURE 1: Lung histopathology photographs. (a)-(d) Light microscope images (HE, $\times 200)$; (e)-(h) electron microscope images; (a) and (e) photographs from the sham-operated group; (b), (c), (f), and (g) photographs from the EP group; (d) and (h) photographs from ShenFu injection group. (b) and (c) Pulmonary arterioles contain erythrocytes, oedema fluid, and inflammatory cells (arrows). (d) Congestion, inflammatory cell infiltration and edema of the lung tissue were considerably alleviated (arrows). (e) normal type II epithelial cells structure was observed in the SHAM group (arrows). (f) Type II epithelial cells are heavily injured, exhibiting necrosis and disintegration (arrows). (g) The structure of the lamellar bodies in type II epithelial cell presents vacuolar change (arrows) inside the cell. (h) The lamellar body of type II epithelial cells shows minor damage in SFI group (arrows).

3.3. The Change of Lung W/D Ratio. The lung W/D ratio was markedly increased in the SA, EP, and SFI groups (6.27 \pm 0.31 , $5.93 \pm 0.29$, and $3.89 \pm 0.27)$ compared with that in the SHAM group $(2.41 \pm 0.21)(P<0.01)$, while the lung $\mathrm{W} / \mathrm{D}$ ratio was significantly decreased in the SFI group compared with the $\mathrm{SA}$ and EP groups at $24 \mathrm{~h}$ after ROSC $(P<0.05)$ (Table 3$)$.
3.4. BALF Total Cell and PMN Counts. As illustrated in Table 4, the post-ROSC group showed a remarkably higher BALF total cell and PMN counts when compared with shamoperated group $(P<0.01)$. Furthermore, the SFI group showed a remarkably lower BALF total cell and PMN counts when compared with SA and EP groups $(P<0.01)$. 
TABLE 3: The chang of lung W/D ratio at $24 \mathrm{~h}$ after ROSC in the SHAM, SA, EP, and SFI groups (mean $\pm \mathrm{SD}$ ).

\begin{tabular}{|c|c|c|c|c|}
\hline & SHAM & SA & $\mathrm{EP}$ & SFI \\
\hline $\begin{array}{l}\text { Lung W/D } \\
\text { ratio }\end{array}$ & $2.41 \pm 0.21$ & $6.27 \pm 0.31^{* *}$ & $5.93 \pm 0.29^{* *}$ & $3.89 \pm 0.27^{*}$ \\
\hline
\end{tabular}

The values represent mean $\pm \mathrm{SE}(n=6){ }^{*} P<0.05$ and ${ }^{* *} P<0.01$ versus SHAM; ${ }^{\mathbf{\Lambda}} P<0.05$ versus EP and SA.

TABLE 4: The change of BALF total cell $\left(\times 10^{7}\right)$ and PMN counts $\left(\times 10^{7}\right)$ at $24 \mathrm{~h}$ after ROSC in the SHAM, SA, EP and SFI groups (mean $\pm \mathrm{SD}$ ).

\begin{tabular}{lcccc}
\hline & SHAM & SA & EP & SFI \\
\hline $\begin{array}{l}\text { BALF total } \\
\text { cell }\end{array}$ & $0.56 \pm 0.11$ & $6.11 \pm 0.65^{*}$ & $7.02 \pm 0.39^{*}$ & $4.33 \pm 0.19^{* \boldsymbol{\Delta}}$ \\
$\begin{array}{l}\text { BALF PMN } \\
\text { counts }\end{array}$ & $0.49 \pm 0.09$ & $4.11 \pm 0.21^{*}$ & $5.66 \pm 0.29^{*}$ & $2.91 \pm 0.31^{* \boldsymbol{\Delta}}$ \\
\hline
\end{tabular}

The values represent mean $\pm \mathrm{SE}(n=6)^{*} P<0.05$ versus SHAM; ${ }^{\mathbf{\Delta}} P<0.05$ versus $\mathrm{EP}$ and $\mathrm{SA}$.

3.5. ELISA Analysis of IL-4 and IFN- $\gamma$ Levels. The results showed that the levels of serum IL-4 were markedly increased at 1/2-24 h after ROSC, while IFN- $\gamma$ and IFN- $\gamma /$ IL- 4 (Th1/Th2) were significantly decreased in the SA, EP, and SFI groups compared with those in the SHAM group $(P<0.05$ or $P<0.01)$ at 6-24 after ROSC. Particularly, the levels of serum IL- 4 at $12 \mathrm{~h}$ were higher than those at all time points after ROSC $(P<0.01)$; however, the levels of serum IFN- $\gamma$ were at $12 \mathrm{~h}$ lower than that at all time points after ROSC $(P<0.01)$ (Figure 2). Furthermore, the levels of lung tissue IL-4 were markedly increased in the SA, EP and SFI groups compared with those in the SHAM group $(P<0.05$ or $P<0.01)$ at $24 \mathrm{~h}$ after ROSC, while IFN- $\gamma$ and IFN- $\gamma / \mathrm{IL}-4$ (Th1/Th2) were significantly decreased, and the levels of lung IL-4 were markedly decreased in the SFI group compared with those in the SA and EP groups $(P<0.05$ or $P<0.01)$ at $24 \mathrm{~h}$ after ROSC, whereas IFN- $\gamma$ and IFN- $\gamma /$ IL- 4 (Th1/Th2) were markedly increased (Figure 2).

3.6. Protein Expressions of GATA-3 and T-bet. As shown in Figure 3, western blot analysis showed that GATA-3 expression progressively increased in the lung tissue $(P<$ 0.05 ), while the expression of T-bet progressively decreased in the SA, EP and SFI groups compared with the SHAM group at $24 \mathrm{~h}$ after ROSC. Furthermore, GATA-3 expression was significantly decreased, while T-bet was significantly increased in the SFI group compared with the EP and SA groups at $24 \mathrm{~h}$ after $\operatorname{ROSC}(P<0.05)$.

3.7. The $m R N A$ Levels of GATA-3 and T-bet. The mRNA levels of the GATA-3 gene were significantly increased $(P<0.01)$, while the mRNA levels of the T-bet gene were significantly decreased in the SA, EP, and SFI groups compared with those in the SHAM group $(P<0.01)$. Furthermore, the mRNA levels of GATA-3 in the SFI group were reduced compared with those in the EP and SA groups $(P<0.05)$ at $24 \mathrm{~h}$ after
ROSC, whereas the mRNA levels of T-bet were increased $(P<0.05)$ in the SFI group (Figure 3$)$.

\section{Discussion}

During CA, a compensatory increase in systemic oxygen extraction occurs, which leads to significantly decreased central or mixed venous oxygen saturation [18]. Inadequate tissue oxygen delivery can persist even after ROSC; accumulated oxygen debt leads to activation of immunologic pathways and systemic inflammation [1], which increases the risk of multiple organ failure [19-21]. The specific structural and functional characteristics of lung tissue make it very vulnerable even after circulation has been successfully restored. In the present study, pigs that underwent $\mathrm{CA}$ all presented with severe post-resuscitation lung injury, manifested by severe histopathological damage, low oxygenation index, high BALF total cell and PMN counts and increased lung W/D ratio. Furthermore, an shift from the Th1 to Th2 response induced by the change in expression levels of the transcription factors GATA-3 and T-bet in the lung tissue occurs in postresuscitation lung injury.

Our results show that SFI group had a better-histopathological change, decreased lung W/D ratio, high oxygenation index, and low BALF total cell and PMN counts compared to the EP and SA groups, suggesting that SFI can reduce postresuscitation lung injury. "SFI decoction" has been commonly used in China for nearly 800 years. SFI is a typical form of SFI decoction for intravenous medication, whose main components include ginsenoside $(0.8 \mathrm{mg} / \mathrm{mL})$ and aconitine $(0.1 \mathrm{mg} / \mathrm{mL})$ [5]. Some studies have also shown that the effects of SFI are based on aconitine properties, supplemented by ginsenoside, which can scavenge free radicals, improve energy metabolism [22], inhibit inflammatory mediators [23], suppress cell apoptosis [5], and alleviate mitochondrial damage [24]. A previous study undertaken in our laboratory showed that SFI attenuated postresuscitation lung injury by inhibiting lung cell apoptosis, and by improving energy metabolism and antioxidant capacity [5].

In actual study, the expression levels of IFN- $\gamma$ and IL-4 depend on the activity of Th1 and Th 2 cells. IFN- $\gamma$, which is generated by activated $\mathrm{T}$ and NK cells, can promote Th 0 cells to differentiate Th1 cells and inhibit the proliferation of Th2 cells [25]. IL-4 can stimulate and activate the proliferation of $\mathrm{B}$ and $\mathrm{T}$ cells, promote the differentiation of Th0 cells into Th2 cells and inhibit the differentiation of Th0 cells into Thl cells. In our study, post-ROSC pigs exhibited a change in their Th1/Th2 cytokine profile in the serum and lung tissue, which was characterized by an increase in IL4 and a decrease in IFN- $\gamma$. During the early postresuscitation period, CA contributes to hemodynamic disorders that cause the systemic release of massive oxygen free radical, lactic acid, and metabolites of arachidonic acid, which could reach the lung tissues by blood circulation and cause ischemia-reperfusion injury. These inflammatory factors are also interactive and make the inflammatory response lose control, which contribute to a lung imbalance of $\mathrm{Th} 1 / \mathrm{Th} 2$. This imbalance promotes lung immune suppression and 


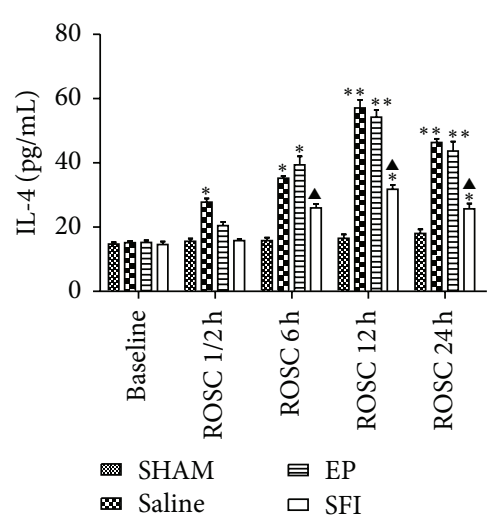

(a)

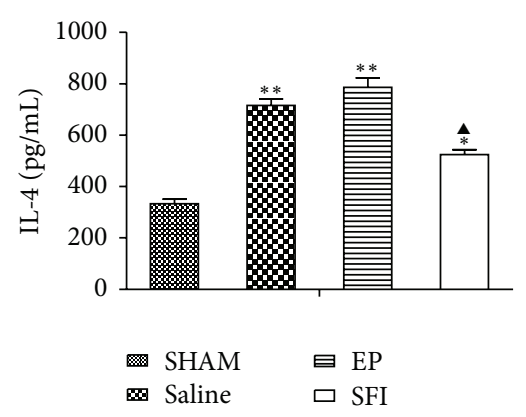

(d)

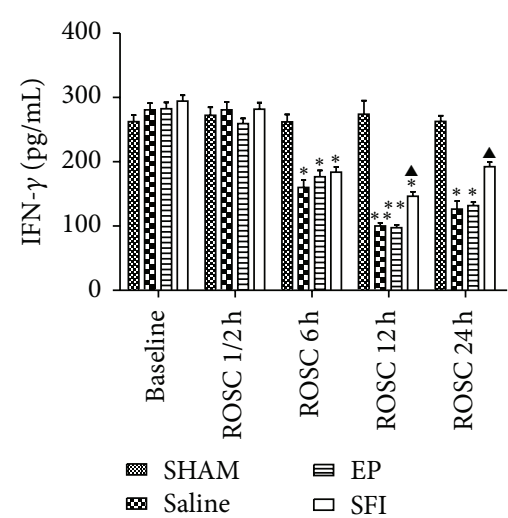

(b)

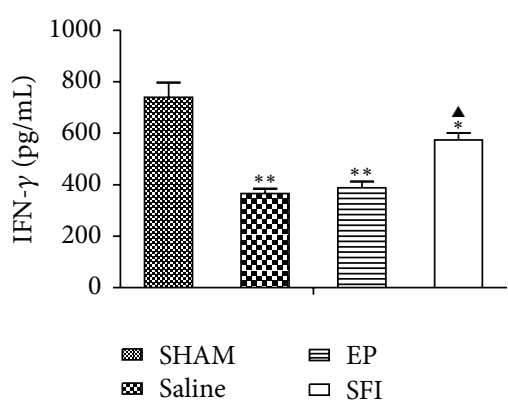

(e)

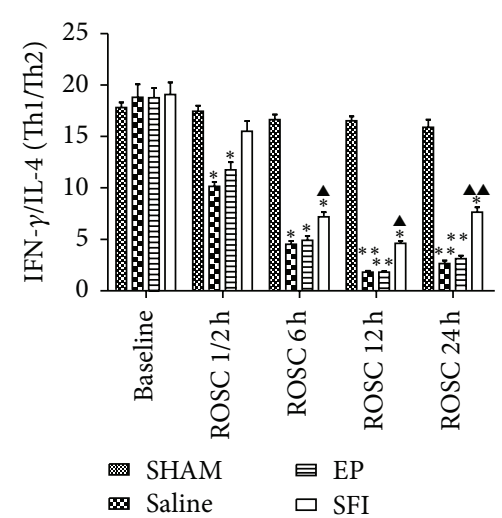

(c)

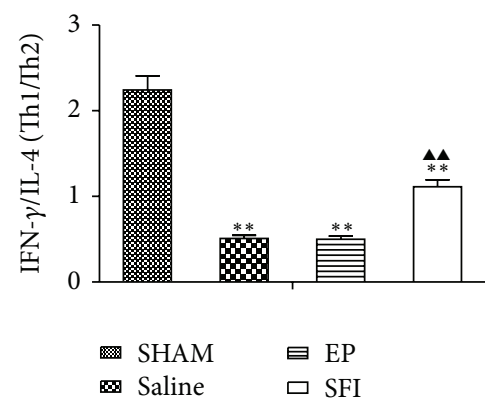

(f)

FIGURE 2: ELISA analysis of (a)-(c) IL-4, IFN- $\gamma$, and IFN- $\gamma /$ IL- 4 levels in the serum; (d)-(f) IL- 4 , IFN- $\gamma$, and IFN- $\gamma /$ IL- 4 levels in the lung tissue: significant difference $\left({ }^{*} P<0.05 ;{ }^{* *} P<0.01\right.$ versus SHAM) between the SHAM group, SA, EP, and SFI group $(n=6)$, ${ }^{\mathbf{\Delta}} P<0.05$, ${ }^{\mathbf{\Delta}} P<0.01$ versus EP and SA.

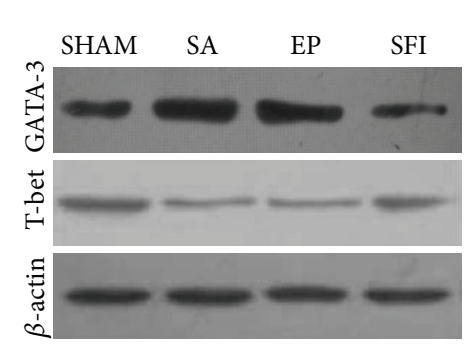

(a)

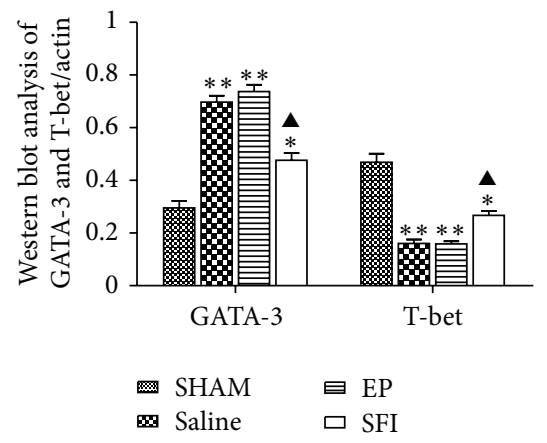

(b)

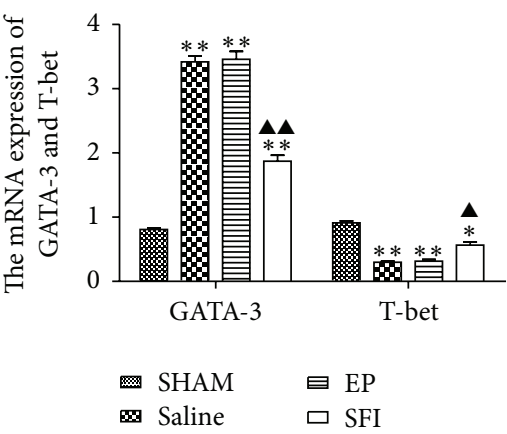

(c)

FIgURE 3: ELISA analysis of IL-4, TNF- $\alpha$, and IFN- $\gamma$ levels in the lung tissue: (a) western blots of GATA-3 and T-bet protein expressions in the lung tissue at $24 \mathrm{~h}$ after ROSC. (b) Western blots of quantification of GATA-3 and T-bet protein levels. (c) mRNA expressions of GATA-3 and T-bet in the lung tissue at $24 \mathrm{~h}$ after ROSC. The values represent mean $\pm \mathrm{SE}(n=6){ }^{*} P<0.05$ and ${ }^{* *} P<0.01$ versus SHAM; ${ }^{\mathbf{4}} P<0.05$, ${ }^{\mathbf{\Lambda}} P<0.01$ versus EP and SA. (one-way repeated-measures ANOVA).

improves development of lung injury. Furthermore, this lung imbalance of Th1/Th2 could increase the risk of multiple organ failure and infection [19], This condition had many features in common with sepsis [20].

In some previous study, the immunomodulatory effects of SFI could (1) reduce the expression of TNF- $\alpha$ to block the vicious circle of inflammatory response, (2) improve systemic microcirculation, prolong the hypoxia tolerance duration, increase blood supply and oxygenation, and promote the release and application of oxygen, (3) inhibit xanthine oxidase directly, protect the SOD activity, and resist lipid peroxidation, (4) ginsenoside could block the cell calcium channel to prevent calcium overload, it could also promote the synthesis of pulmonary surfactant and improve oxygenation, (5) promote the liver detoxifying function to reduce the endotoxin caused lung injury $[14,15,26]$. Furthermore, a previous study 
demonstrated that ginsenoside could stimulate immune function, improve both specific and nonspecific immunity, and influence both humoral immunity and cell immunity. They showed that total ginseng soap could promote the biosynthesis of proteins [27]. fuzi could also improve immune function by enhancing the adrenocortical system [26]. Our results also show that SFI group could improve lung immune function by modulating the Th1/Th2 imbalance. Thus, SFI could reduce postresuscitation lung injury and improve lung immune function by regulating lung imbalance of Th1/Th2.

GATA-3, a member of the GATA family of zinc-finger transcription factors, has been identified as a key regulator of Th2 cell development [28]. In contrast, T-bet, a member of the T-box family of transcription factors, is a key controller of Th1 cell differentiation [28]. GATA3 specifies Th2 lineage commitment by inducing lineagerestricted target genes, such as IL-4, whereas T-bet specifies Th1 lineage commitment by inducing genes for IFN- $\gamma[28$, 29]. Correspondingly, a increase in GATA- 3 could lead to IL4 production, whereas T-bet leads to suppression of IFN- $\gamma$ production. In the present study, both the protein expression and mRNA levels of GATA-3 were markedly reduced, while T-bet was significantly increased in the lung tissue of pigs in the SFI group compared with that in the EP groups. SFI can therefore promote the secretion of the Thl cytokine IFN$\gamma$ by upregulating the expression of T-bet, and inhibit the Th2 cytokine IL- 4 by downregulating GATA-3 in the lung tissue. Thus, SFI can modulate the lung imbalance of T-bet and GATA-3 expression levels and correct and restore the balance of lung Thl and Th2 immune cells. Furthermore, our results show that SFI has certain advantages compared with EP and SA, not only improving lung histopathological change and reducing lung injury, but also modulating lung Th1/Th2 imbalances by regulating changes in the expression of the transcription factors GATA-3 and T-bet in the lung tissue.

\section{Conclusion}

Our results demonstrate that SFI could improve lung immune function and protect from postresuscitation lung injury by modulating Th1/Th2 cells imbalances in the lung tissue compared with EP and SA.

\section{Conflict of Interests}

The authors state that they have no commercial associations or sources of support that might pose a conflict of interests.

\section{Acknowledgments}

The authors sincerely thank Professors Ping Wei and Yilin Sun, who provided technical help for the analysis of lung specimens using electron microscopy.

\section{References}

[1] A. Karimova and D. J. Pinsky, "The endothelial response to oxygen deprivation: biology and clinical implications," Intensive Care Medicine, vol. 27, no. 1, pp. 19-31, 2001.
[2] W. C. Schoemaker, P. L. Appel, and H. B. Kram, “Tissue oxygen debt as a determinant of lethal and nonlethal postoperative organ failure," Critical Care Medicine, vol. 16, no. 11, pp. 11171120, 1988.

[3] M. D. Schwartz, J. E. Repine, and E. Abraham, "Xanthine oxidase-derired oxygen radicals increase lung cytokine expression in mice subjected to hemorrhagic shock," American Journal of Respiratory Cell and Molecular Biology, vol. 12, pp. 434-440, 1995.

[4] Y. Huang, H. Rabb, and K. L. Womer, "Ischemia-reperfusion and immediate T cell responses," Cellular Immunology, vol. 248, no. 1, pp. 4-11, 2007.

[5] M. Y. Zhang, X. F. Ji, S. Wang, and C. S. Li, "Shen-Fu injection attenuates postresuscitation lung injury in a porcine model of cardiac arrest," Resusciatation, vol. 83, no. 9, pp. 1152-1158, 2012.

[6] A. K. Abbas, K. M. Murphy, and A. Sher, "Functional diversity of helper T lymphocytes," Nature, vol. 383, no. 6603, pp. 787-793, 1996.

[7] R. S. Liblau, S. M. Singer, and H. O. McDevitt, "Th1 and Th2 $\mathrm{CD}^{+} \mathrm{T}$ cells in the pathogenesis of organ-specific autoimmune diseases," Immunology Today, vol. 16, no. 1, pp. 34-38, 1995.

[8] D. H. Zhang, L. Cohn, P. Ray, K. Bottomly, and A. Ray, "Transcription factor GATA-3 is differentially expressed in murine Th1 and Th2 cells and controls Th2-specific expression of the interleukin-5 gene," Journal of Biological Chemistry, vol. 272, no. 34, pp. 21597-21603, 1997.

[9] L.-F. Huang, Y.-M. Yao, J.-F. Li et al., "Reduction of plasma gelsolin levels correlates with development of multiple organ dysfunction syndrome and fatal outcome in burn patients," PLoS One, vol. 6, no. 10, Article ID e25748, 2011.

[10] M. Tan, J.-C. Zhu, J. Du, L.-M. Zhang, and H.-H. Yin, "Effects of probiotics on serum levels of Th1/Th2 cytokine and clinical outcomes in severe traumatic brain-injured patients: a prospective randomized pilot study," Critical Care, vol. 15, no. 6, article R290, 2011.

[11] X. Cheng, Y. H. Liao, H. Ge et al., “Th1/Th2 functional imbalance after acute myocardial infarction: coronary arterial inflammation or myocardial inflammation," Journal of Clinical Immunology, vol. 25, no. 3, pp. 246-253, 2005.

[12] J. Szkodzinski, B. Hudzik, M. Osuch et al., "Serum concentrations of interleukin-4 and interferon-gamma in relation to severe left ventricular dysfunction in patients with acute myocardial infarction undergoing percutaneous coronary intervention," Heart and Vessels, vol. 26, no. 4, pp. 399-407, 2011.

[13] W. F. Carson, T. Ito, M. Schaller, K. A. Cavassani, S. W. Chensue, and S. L. Kunkel, "Dysregulated cytokine expression by $\mathrm{CD} 4^{+}$ $\mathrm{T}$ cells from post-septic mice modulates both Th1 and Th2mediated granulomatous lung inflammation," PLoS One, vol. 6, no. 5, Article ID e20385, 2011.

[14] Z. Y. Xia, L. Wang, X. J. Chen et al., "Protective effect of Shenfu injection intestinal mucosa during hemorrhagic shock-reperfusion in rabbits," Chinese Journal of Critical Care Medicine, vol. 21, no. 8, pp. 447-448, 2001.

[15] Y. H. Ai, L. Peng, and L. N. Zhang, "Protective effect of Shenfu injection on endotoxin induced acute lung injury," Chinese Journal of Critical Care Medicine, vol. 26, no. 4, pp. 285-286, 2006.

[16] W. Gu, C. S. Li, W. P. Yin, Z. J. Guo, X. M. Hou, and D. Zhang, "Apoptosis is involved in the mechanism of postresuscitation myocardial dysfunction in a porcine model of cardiac arrest," American Journal of Emergency Medicine, vol. 30, no. 9, pp. 2039-2045, 2012. 
[17] W. Gu, C. Li, W. Yin, Z. Guo, X. Hou, and D. Zhang, "ShenFu injection reduces postresuscitation myocardial dysfunction in a porcine model of cardiac arrest by modulating apoptosis," Shock, vol. 38, no. 3, pp. 301-306, 2012.

[18] E. P. Rivers, G. B. Martin, H. Smithline et al., “The clinical implications of continuous central venous oxygen saturation during human CPR," Annals of Emergency Medicine, vol. 21, no. 9, pp. 1094-1101, 1992.

[19] E. L. Cerchiari, P. Safar, E. Klein, and W. Diven, "Visceral, hematologic and bacteriologic changes and neurologic outcome after cardiac arrest in dogs. The visceral post-resuscitation syndrome," Resuscitation, vol. 25, no. 2, pp. 119-136, 1993.

[20] C. Adrie, M. Adib-Conquy, I. Laurent et al., "Successful cardiopulmonary resuscitation after cardiac arrest as a "sepsis-like" syndrome," Circulation, vol. 106, no. 5, pp. 562-568, 2002.

[21] C. Adrie, I. Laurent, M. Monchi, A. Cariou, J. F. Dhainaou, and C. Spaulding, "Postresuscitation disease after cardiac arrest: a sepsis-like syndrome?" Current Opinion in Critical Care, vol. 10, no. 3, pp. 208-212, 2004.

[22] J. Tian, F. Fu, M. Geng et al., "Neuroprotective effect of 20(S)ginsenoside $\mathrm{Rg}_{3}$ on cerebral ischemia in rats," Neuroscience Letters, vol. 374, no. 2, pp. 92-97, 2005.

[23] W. Li, L. Li, Y. Q. Chu et al., "Ginsenoside decreases expression of phosphorylated ERK and cytokine content in THP-1 cells induced by $\beta$-amyloid," Basic and Clinical Medicine, vol. 26, pp. 362-365, 2006.

[24] J. Tian, S. Zhang, G. Li, Z. Liu, and B. Xu, "20(S)-Ginsenoside $\mathrm{Rg}_{3}$, a neuroprotective agent, inhibits mitochondrial permeability transition pores in rat brain," Phytotherapy Research, vol. 23, no. 4, pp. 486-491, 2009.

[25] J. G. Williams, G. J. Jurkovich, and R. V. Maier, "Interferon$\gamma$ : a key immunoregulatory lymphokine," Journal of Surgical Research, vol. 54, no. 1, pp. 79-93, 1993.

[26] F. F. Han and J. H. Lin, "Influences of Shenfu Injection on immune functions in patients with respiratory failure," Zhongguo Zhong Xi Yi Jie He Za Zhi, vol. 6, no. 11, pp. 505-506, 1999.

[27] X. Tian, D. M. Gou, and H. J. Cai, "Effect of shenfu injection on the erythrocyte immune function of patients undergoing cardiopulmonary bypass," Zhongguo Zhong Xi Yi Jie He Za Zhi, vol. 31, no. 11, pp. 1471-1473, 2011.

[28] W. P. Zheng and R. A. Flavell, "The transcription factor GATA-3 is necessary and sufficient for Th2 cytokine gene expression in CD4 T cells," Cell, vol. 89, no. 4, pp. 587-596, 1997.

[29] S. J. Szabo, S. T. Kim, G. L. Costa, X. Zhang, C. G. Fathman, and L. H. Glimcher, "A novel transcription factor, T-bet, directs Th1 lineage commitment," Cell, vol. 100, no. 6, pp. 655-669, 2000. 


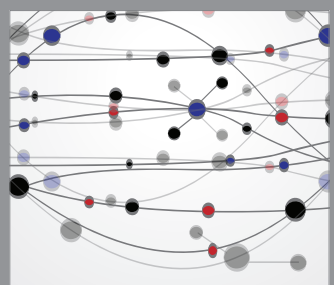

The Scientific World Journal
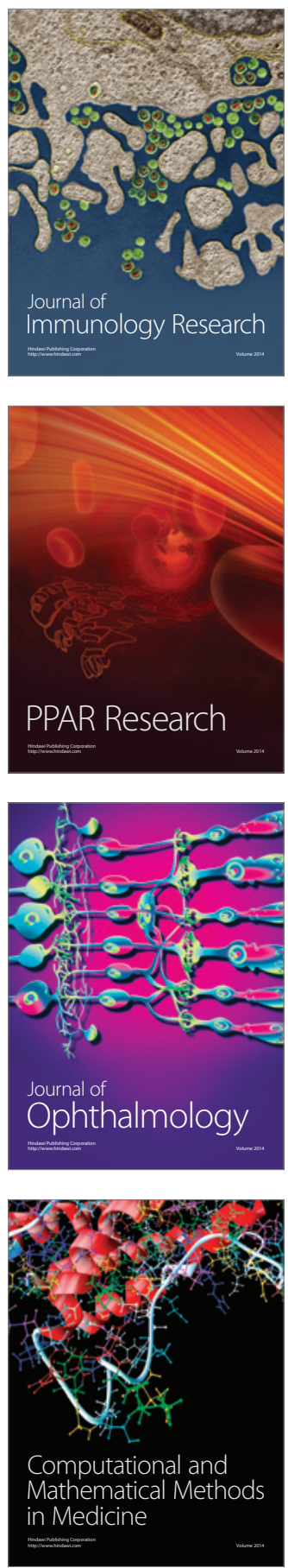

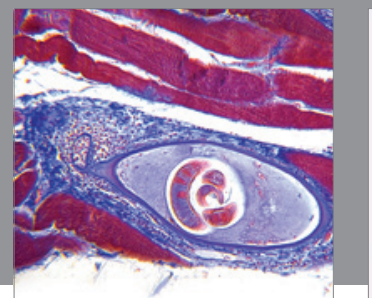

Gastroenterology

Research and Practice
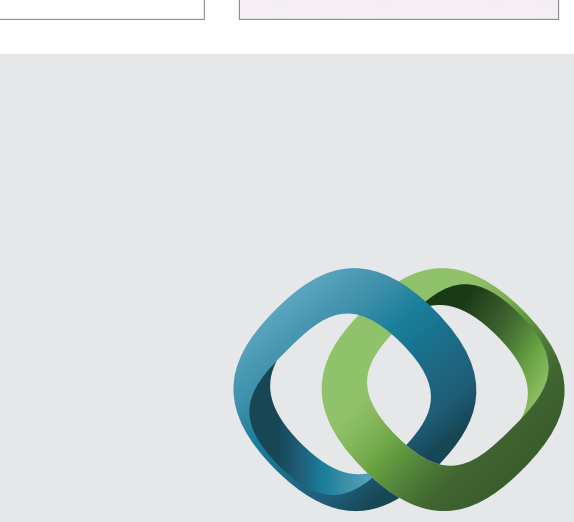

\section{Hindawi}

Submit your manuscripts at

http://www.hindawi.com
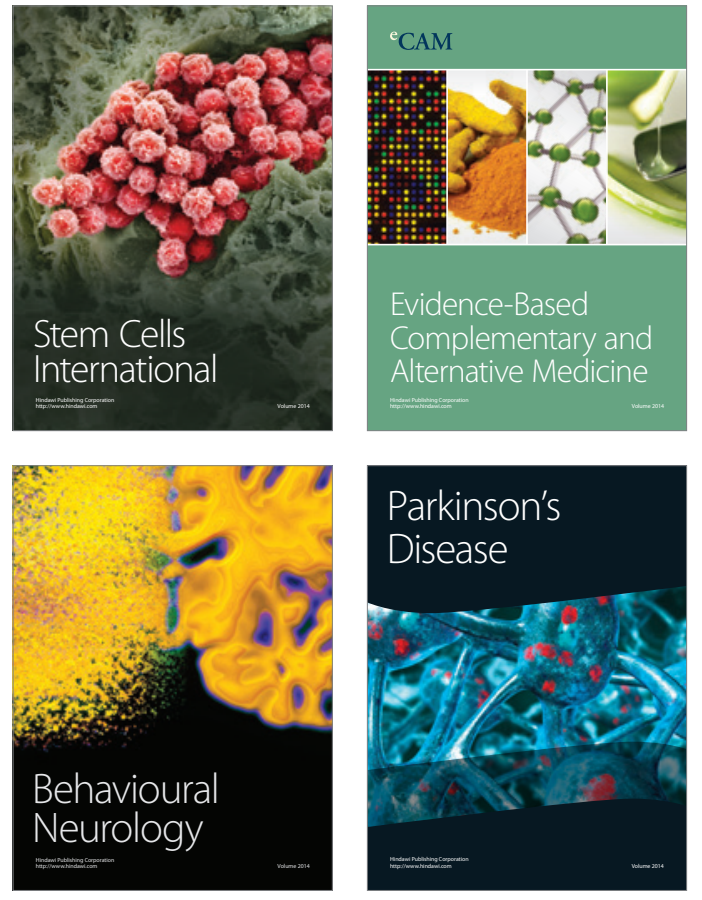
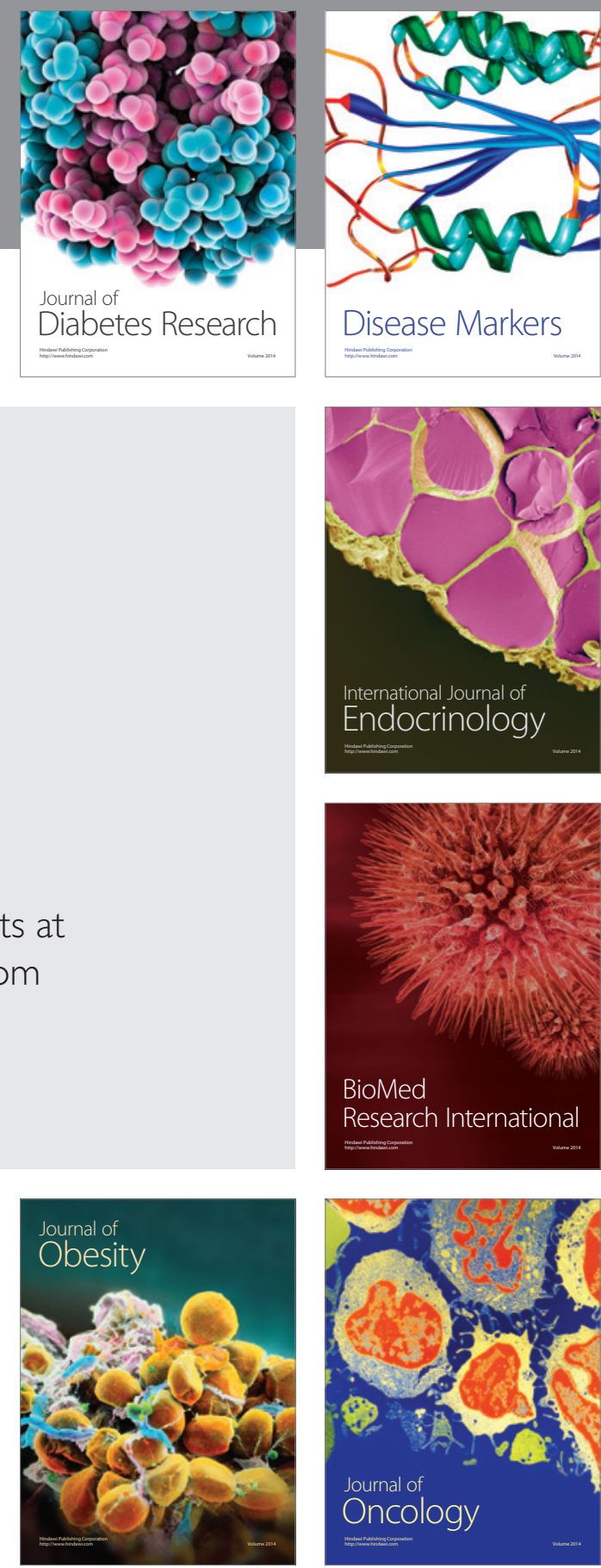

Disease Markers
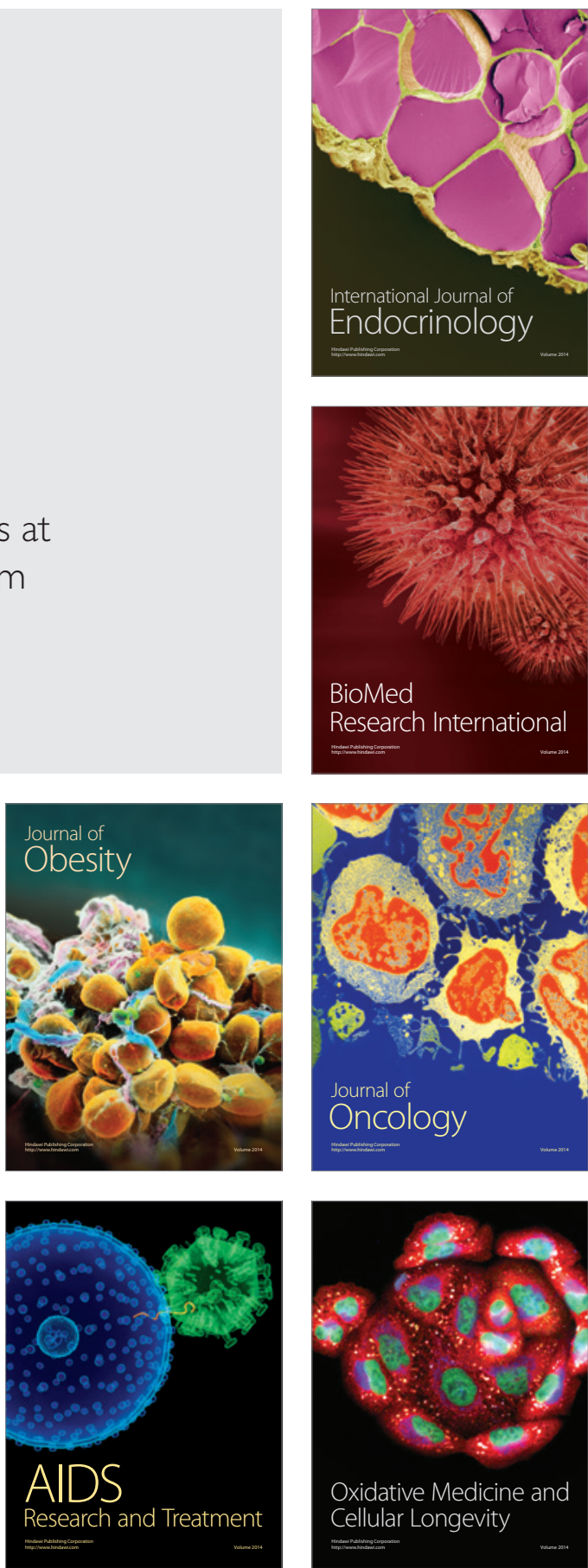